\title{
Influencia de la integración docente asistencial en la calidad de los servicios de salud del primer nivel de atención y en la formación médica en la Universidad Nacional San Antonio Abad del Cusco Influence of teaching-health care integration on the quality of first level health services and medical education at San Antonio Abad del Cusco National University
}

\author{
Santiago Máximo Saco-Méndez¹, Salomón Zavala-Sarrio² \\ ${ }^{1}$ Profesor Principal, Facultad de Medicina - Universidad Nacional de San Antonio Abad del Cusco \\ ${ }^{2}$ Profesor Principal, Facultad de Medicina - Universidad Nacional Mayor de San Marcos
}

\section{Correspondencia}

\section{Santiago Saco-Méndez} santisac@gmail.com

Recibido: 13 de febrero 2017

Aceptado: 5 de junio 2017

El Dr. Santiago Saco declara ser Profesor de la Universidad Nacional San Antonio Abad del Cusco hasta la actualidad.

El Dr. Santiago Saco participó en la concepción, diseño, desarrollo de la investigación, y redacción. El Dr. Salomón Zavala participó en el análisis e interpretación de datos y redacción del artículo.

Tesis presentada para obtener el grado académico de Doctor en Medicina del Dr. Santiago Saco Méndez.

Conflictos de interés: Ninguno. Fuente de financiamiento: Propio.

Citar como: Saco Méndez SM, Zavala Sarrio S. Influencia de la integración docente asistencial en la calidad de los servicios de salud del primer nivel de atención y en la formación médica en la Universidad Nacional San Antonio Abad del Cusco. An Fac med. 2017;78(3): 309-314 DOI: http://dx.doi.org/10.15381/anales.v78i3.13766
An Fac med. 2017;78(3):309-314 / http://dx.doi.org/10.15381/anales.v78i3.13766

Resumen

Introducción. La Organización Panamericana de la Salud (OPS) promueve la formación médica con énfasis en el componente social, a través de la integración docente asistencial (IDA) definida como el proceso de articulación entre instituciones de servicios de salud e instituciones de educación, para contribuir a mejorar las condiciones de vida de la colectividad, mediante la prestación de servicios adecuados a las necesidades reales de la población. Objetivo. Determinar si la integración docente asistencial influye en la mejora de la calidad de los servicios de salud de la comunidad y en la formación integral de los médicos. Diseño. Estudio observacional, descriptivo. Lugar. Facultad de Medicina de la Universidad Nacional de San Antonio Abad del Cusco y establecimientos de salud del primer nivel de atención en comunidades de extrema pobreza del Cusco, Perú. Participantes. Usuarios de los establecimientos de salud, familias de las comunidades, trabajadores de los establecimientos de salud, autoridades locales, y estudiantes internos de medicina. Intervenciones. Aplicación de encuesta de satisfacción a pacientes en relación a la atención recibida en los establecimientos y comunidades donde se implementó la IDA; así mismo, encuestas de conocimientos, actitudes y prácticas. Resultados. Las comunidades donde se implementó la IDA mostraron mayor satisfacción por los servicios recibidos, en $48 \%$ de los usuarios. Las familias (64\%), los trabajadores de los establecimientos de salud (95\%), así como las autoridades locales (86\%) valoraron como bueno el trabajo realizado por los estudiantes de medicina a través del internado rural. Los estudiantes valoraron el internado rural como contribución a su formación, en $81 \%$. Conclusiones. La IDA contribuyó a mejorar la situación de salud de las comunidades intervenidas, la calidad de los servicios en los establecimientos de salud, e influyó en la formación y desempeño del futuro médico.

Palabras clave. Organización Mundial de la Salud; Servicios de Integración Docente Asistencial; Servicios de Salud; Educación Médica; Satisfacción de Paciente

Abstract

Introduction: The Pan American Health Organization (PAHO) promotes medical education with emphasis on the social component, through teaching care integration (TCl) defined as the process of articulation between health service and education institutions to contribute to improving the living conditions of the community by provision of adequate services to the real needs of the population. Objective: To determine whether if the teaching care integration influences in improving the quality of health services in the community and in the integral training of physicians. Design: Observational, descriptive study. Setting: Faculty of Medicine of San Antonio Abad del Cusco National University and first level of health attention establishments in extreme poverty communities of Cusco, Peru. Participants: Users of health facilities, community families, health facility workers, local authorities, and internal medicine students. Interventions: Application of patients' satisfaction survey to the care received in the establishments and communities where the teaching care integration was implemented; also surveys on knowledge, attitudes, and practices were applied. Results: The communities where the $\mathrm{TCl}$ was implemented showed greater satisfaction with the services received, in $48 \%$ of the users. Families (64\%), health care workers (95\%), and local authorities $(86 \%)$ valued the work performed by the medical students through the rural intership as good. The students valued the rural boarding school as a contribution to their training in $81 \%$. Conclusions: The teaching care integration contributed to improving health and quality of health services in the communities, and influenced the training and performance of the future physicians.

Keywords: Pan American Health Organization; Teaching Care Integration Services; Health Services; Medical Education; Patient Satisfaction. 


\section{INTRODUCCIÓN}

La Organización Panamericana de la Salud (OPS), en el informe final del grupo reunido en Bahía sobre requisitos mínimos para la creación de escuelas de medicina, concluyó que el fin último del sistema de formación de recursos humanos para la salud no es formar profesionales, sino mejorar la salud de la población (1) Esta afirmación constituye una visión fundamental que debe orientar la formación médica. Así, desde los años 50 surge en las universidades latinoamericanas, con el apoyo de la OPS, un debate sobre las características de la enseñanza de la medicina, proponiéndose cambios para su innovación y adecuación a las necesidades locales. Dentro de este proceso de análisis, crítica y pedidos de reformas en la enseñanza médica, surge el cuestionamiento al modelo flexneriano de la enseñanza de la medicina, que fue introducido en las universidades latinoamericanas a mediados del siglo pasado; este modelo pone énfasis en la enseñanza en los laboratorios y prácticas dentro de los hospitales. En contraposición, surge el movimiento 'preventivista', en los años cincuenta, que redescubre la máxima de Virchow por la cual, 'la medicina es una ciencia social', emprendiendo una constante lucha para ir incorporando en la educación médica aspectos sociales, epidemiológicos y de salud pública. Paralelo a ello, propone que la enseñanza de la medicina no solo debe realizarse en laboratorios y en los hospitales, sino también en la comunidad y en los servicios de salud del primer nivel de atención, bajo el énfasis de la multicausalidad del proceso salud-enfermedad.

En 1978, la Conferencia Internacional sobre la Atención Primaria de la Salud estableció como uno de los principales objetivos sociales de los gobiernos que todos los pueblos del mundo alcancen en el año 2000 un nivel de vida social y económicamente productivo. La atención primaria de la Salud es la clave para alcanzar esa meta como parte del desarrollo conforme al espíritu de justicia social $^{(2)}$. En 1988 y 1993 se llevaron a cabo la Primera y Segunda Conferencia Mundial sobre Educación Médica, ambas en Edimburgo, sobre el tema del cambio de la práctica médica y su influencia sobre la educación médica, considerando el avance tecnológico y el imperativo de equidad. Señalaron en su declaración que el objetivo de la educación médica es producir médicos que promuevan la salud. Dicho objetivo no se está logrando, a pesar del progreso de la ciencia biomédica, por la carencia de esfuerzos por producir mayor conciencia social en las escuelas de medicina ${ }^{1}$

Durante años, los ministerios de salud y muchas universidades latinoamericanas, con el apoyo de la OPS y la OMS, vienen buscado mecanismos de articulación entre la formación del personal de salud y las necesidades de los servicios de salud, con miras a que estos respondan a las necesidades de salud de la población. Así, las tendencias de la educación médica plantean que la formación del profesional de la salud debe darse en la realidad concreta y a nivel local, donde se da el proceso salud-enfermedad. Se propone, de ese modo, la Integración Docente Asistencial (IDA), como el proceso de articulación entre instituciones de servicios de salud e instituciones de educación, para contribuir a mejorar las condiciones de vida de la colectividad mediante la prestación de servicios adecuados a las necesidades reales de la población, la producción de conocimientos y la formación de recursos humanos necesarios en un determinado contexto de la práctica de servicios de salud y de enseñanza ${ }^{(3)}$.

Siguiendo las nuevas tendencias en educación médica y las políticas de salud, y la Declaración de Alma Ata, la Facultad de Medicina Humana de la Universidad Nacional de San Antonio Abad del Cusco (FMH-UNSAAC), en su proyecto de creación, planteó cambios en la educación médica, incorporando la enseñanza teórica y práctica de la Medicina Preventiva Social, la Medicina Comunitaria, la interdisciplinariedad, así como el uso de los diversos servicios de salud, que permitan la incorporación del estudiante a los diferentes niveles de atención y el aprendizaje en el trabajo ${ }^{(4)}$. De acuerdo a los lineamientos antes mencionados, desde 1980 hasta la actualidad la FMH-UNSAAC implementa la IDA en la formación médica. Así, los estudiantes desde los primeros años hacen prácticas en los centros de salud urbano-marginales y rurales del
Cusco y llevan cursos acordes con dicho enfoque, como quechua y medicina tradicional, incluyéndose estas actividades en los cursos clínicos (medicina, pediatría, ginecoobstetricia y cirugía) y no solo en los servicios hospitalarios del tercer nivel. En el penúltimo año, los estudiantes realizan el internado rural, durante 3 meses, en un establecimiento de salud del área rural, incorporándose el interno en el trabajo del equipo de salud del establecimiento $(5,6)$. Los estudiantes quedan bajo la tutoría de los médicos del establecimiento y son supervisados mensualmente por los docentes de la facultad así como por las autoridades locales donde se realiza este tipo de internado. Para ello, la UNSAAC, gestionó y logró establecer una serie de convenios con el Ministerio de Salud a nivel nacional y regional para garantizar la ejecución de la IDA. De esa manera, la presencia de los estudiantes y docentes constituye un elemento dinamizador de las actividades de salud, sobre todo de la promoción y prevención de la salud, contribuyendo a mejorar la calidad de los servicios de salud, tales como: atenciones de salud, atención de las estrategias sanitarias, las visitas domiciliarias de los enfermos, la inspección de los manantes de agua, de las letrinas construidas, el trabajo que realizan en los centros educativos con los niños, la implementación de los rincones de aseo escolar, la desparasitación y despiojamiento de los niños, las charlas educativas con las madres de familia, la cloración de los tanques de agua, concurso de la casa más limpia y ordenada, el equipamiento de botiquines comunales, y la implementación de los huertos familiares. Las actividades mencionadas fueron realizadas por los alumnos, los comuneros y el centro de salud en las diversas comunidades. Por otro lado, se realizaron investigaciones respecto a los problemas de salud más frecuentes en las comunidades.

Los fines de la implementación de la IDA en la FMH-UNSAAC son:

1. Conocer la realidad socio cultural, económica y de salud de comunidades andinas y amazónicas de extrema pobreza, futuro primer escenario de trabajo del médico joven, y contribuir a mejorar sus condiciones de salud y de vida. 
2. Conocer la dinámica de trabajo de un establecimiento del primer nivel de atención de salud, y cómo se gestiona este establecimiento para el apoyo en el desarrollo de sus actividades.

3. Conocer la patología de estas zonas, y conocer y aplicar las estrategias para su promoción, prevención, curación y rehabilitación, aplicando las estrategias de salud del MINSA.

4. Conocer e interpretar los factores determinantes de la salud-enfermedad de estas zonas.

5. Conocer, y valorar los patrones culturales (idioma, costumbres), su cosmovisión de salud-enfermedad y sus prácticas médicas con la que la comunidad busca resolver sus problemas de salud.

6. Conocer la dinámica de la organización comunal y de su participación en la solución de sus problemas de salud.

7. Realizar investigaciones sobre problemas prioritarios de salud.

8. Conocer y aplicar las diversas metodologías de capacitación de poblaciones.

9. Realizar actividades de medicina legal.

10. Desarrollar actividades de saneamiento básico.

11. Tener una verdadera formación médica integral.

El objetivo de la presente investigación fue determinar la influencia de la IDA en la calidad de los servicios de salud del primer nivel de atención en comunidades rurales donde los estudiantes de medicina realizan el internado rural; además, determinar la influencia de la IDA en la formación de los médicos en la FMHUNSAAC.

\section{MÉTODOS}

Se realizó un estudio observacional, descriptivo, seleccionándose para ello mediante muestreo intencional nueve establecimientos de salud del primer nivel de atención de comunidades de extrema pobreza en Cusco, seis de ellos donde se implementó la IDA. Para cada establecimiento de salud se seleccionaron usuarios mediante muestreo estratificado, con la finalidad de realizar encuestas de satisfacción de la atención recibida.

De igual manera, se hizo una selección de familias por muestreo aleatorio en comunidades que presentan mayores problemas sanitarios, de distintas ubicaciones geográficas y condiciones socioeconómicas, donde laboran los estudiantes. A las familias se realizó la encuesta sobre sus opiniones y las ventajas de la presencia de dichos estudiantes así como el nivel de conocimientos, prácticas y actitudes que tienen las familias campesinas sobre sus problemas más frecuentes de salud.

Por otro lado, se evaluaron los resultados sanitarios de los proyectos comunitarios en salud que han sido desarrollados por la universidad con la participación de los estudiantes.

También se realizó una encuesta tanto a los trabajadores de los establecimientos de salud, como a las autoridades locales y de salud, y a los estudiantes que habían realizado el internado rural, con la finalidad de conocer su opinión de la implementación de la IDA.

La presente investigación, tuvo en cuenta los aspectos éticos recomendados sobre investigaciones con seres humanos; para ello, los participantes del estudio fueron informados sobre el mismo, dando su consentimiento informado para poder participar del estudio. La información proporcionada fue manejada con la confidencialidad del caso.

\section{RESULTADOS}

De la revisión de los documentos en los archivos de los establecimientos y de la facultad se encontró que los estudiantes realizan diversas actividades integrales de salud, que van desde atenciones en consultorios, emergencias, programas, tópico, y extramural (realizan visitas domiciliarias), capacitaciones en las escuelas, con las madres y la comunidad en general, lo que les permite conocer y actuar sobre los problema de la salud co- munal en forma integral, repercutiendo en la mejora de las actividades del establecimiento de salud y por ende de la comunidad. Estas actividades fueron establecidas en el plan de trabajo a través de la ficha de Programación de Actividades para el Internado Rural.

Las familias encuestadas fueron de las localidades donde se implementó la IDA: Usi Alto en Quiquijana, Machaca en Ccatcca, Patapallpa Baja en Ocongate, Tiracancha en Huancarani, Espinguni en Paucartambo, y Atalaya en Pilcopata, haciendo un total de 118 familias. Y también, en tres comunidades donde no se implementó la IDA: Pallata en Ollantaytambo, Molle Molle en Huanoquite y Sicre en Huyro, con un total de 70 familias.

La edad promedio de los jefes de familia encuestados fue de 30 años, 70\% casados y con un promedio de hijos menor a 4. El nivel de conocimientos sobre la salud materna, el uso de anticonceptivos, los problemas y cuidados de la gestación, lactancia materna exclusiva, así como los problemas sanitarios en niños (inmunizaciones, enfermedades diarreicas agudas infecciones respiratorias agudas) fue mayor en las familias donde se implementó la IDA. De igual manera, el nivel de conocimientos respecto a las medidas higiénicas como el lavado de manos, cepillado de dientes, y el uso de letrinas fue mayor en las familias donde se implementó la IDA.

La encuesta de satisfacción de usuarios en el establecimiento de salud se realizó en las nueve comunidades. Participaron pobladores de ambos sexos por igual, con una edad promedio de 29 años, promedio de 3 hijos, y con niveles educativos de nivel secundario (42\%) y nivel primario (40\%); el $90 \%$ de ellos era quechua hablante. Respecto a la encuesta de satisfacción, el 90\% de los encuestados consideró que los servicios del MINSA son accesibles en cuanto a distancia y horarios de atención; $68 \%$ que los trabajadores del establecimiento se comunicaban bien en quechua. El 70\% de usuarios en quienes se implementó la IDA consideró que los trabajadores de salud respetan la medicina tradicional. Asimismo, el $47 \%$ de los encuestados pensaba que el trato fue más amable en los estableci- 
mientos donde se implementó la IDA versus el $26 \%$ donde no se implementó. Un $46 \%$ de los encuestados consideró que el establecimiento de salud les resuelve sus problemas en los establecimientos donde se implementó la IDA, versus un $20 \%$ en los establecimientos donde no se implementó. Además, respecto a la percepción de los campesinos de que si su problema de salud es entendido por las enfermeras y los médicos, los campesinos donde se implementó IDA consideraron que en $57 \%$ su problema fue entendido completamente; solo $34 \%$ de los campesinos donde no se implementó IDA señaló que su problema es entendido por los profesionales de la salud.

Sobre la satisfacción general del servicio, los campesinos de las comunidades donde se implementó la IDA, el $48 \%$ se encontraron más satisfechos con la atención recibida; mientras que en las comunidades donde no se implementó la IDA, el $32 \%$ se mostró satisfecho.

La opinión por parte de los pobladores de las comunidades donde se imple- mentó la IDA, respecto a las actividades integrales de salud, el 64\% las consideró como buenas, y $20 \%$ como muy bueno el trabajo realizado por los estudiantes.

Respecto a la opinión de los trabajadores de los establecimientos de salud donde se implementó la IDA, la mayoría (95\%) la consideró favorable y oportuna la participación de los estudiantes de medicina en las actividades asistenciales y preventivas promocionales del establecimiento.

Respecto a la opinión de las autoridades, se realizó una encuesta dirigida a las autoridades (alcaldes, promotores de salud, profesores, directores de centros educativos, autoridades de la DIRESA) del distrito donde funcionan los establecimientos donde se implementó la IDA. El $100 \%$ de encuestados tenía conocimiento del trabajo de los internos de medicina en los establecimientos, el 75\% información que dichos internos son supervisados por profesores de la UNSAAC, el $86 \%$ consideró como buena la presencia de los internos en el establecimiento de sa- lud. Más del $90 \%$ de los encuestados señaló que los internos tienen trato amable con los pacientes, con las autoridades, y reconocieron la dedicación de los mismos con su trabajo diario en lo referido a las actividades educativas y promoción de la salud comunitaria.

Se realizó una encuesta a 171 alumnos egresados de la FMH-UNSAAC que habían realizado el internado rural. De ellos, la mayoría tenía por debajo de los 30 años de edad y con padres profesionales, datos coincidentes con lo previamente por Brito y col. ${ }^{(7)}$ y por Alarcón, a nivel de Lima Metropolitana ${ }^{(8)}$. La mayoría de los encuestados consideró adecuada la preparación que tuvieron en la Facultad así como de mucho interés el internado rural respecto a las demás rotaciones; así, 81\% valoró el internado rural como bueno y excelente. Las actividades y aspectos de mejora en la formación médica, realizado por los encuestados, se encuentran descritas en las figuras 1 y 2 .

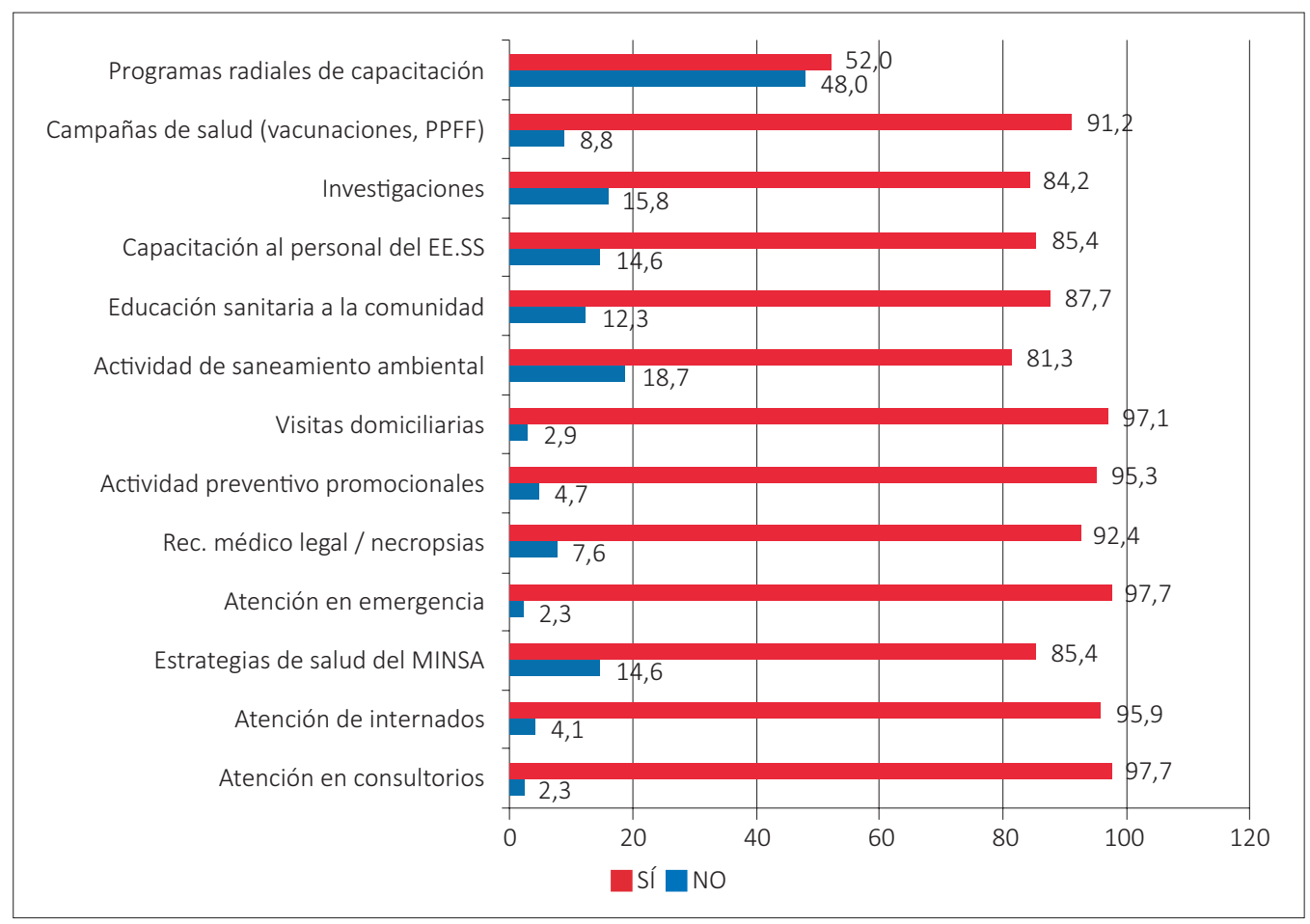

Figura 1. Actividades realizadas en el internado rural por parte de los egresados FMH-UNSAAC. 


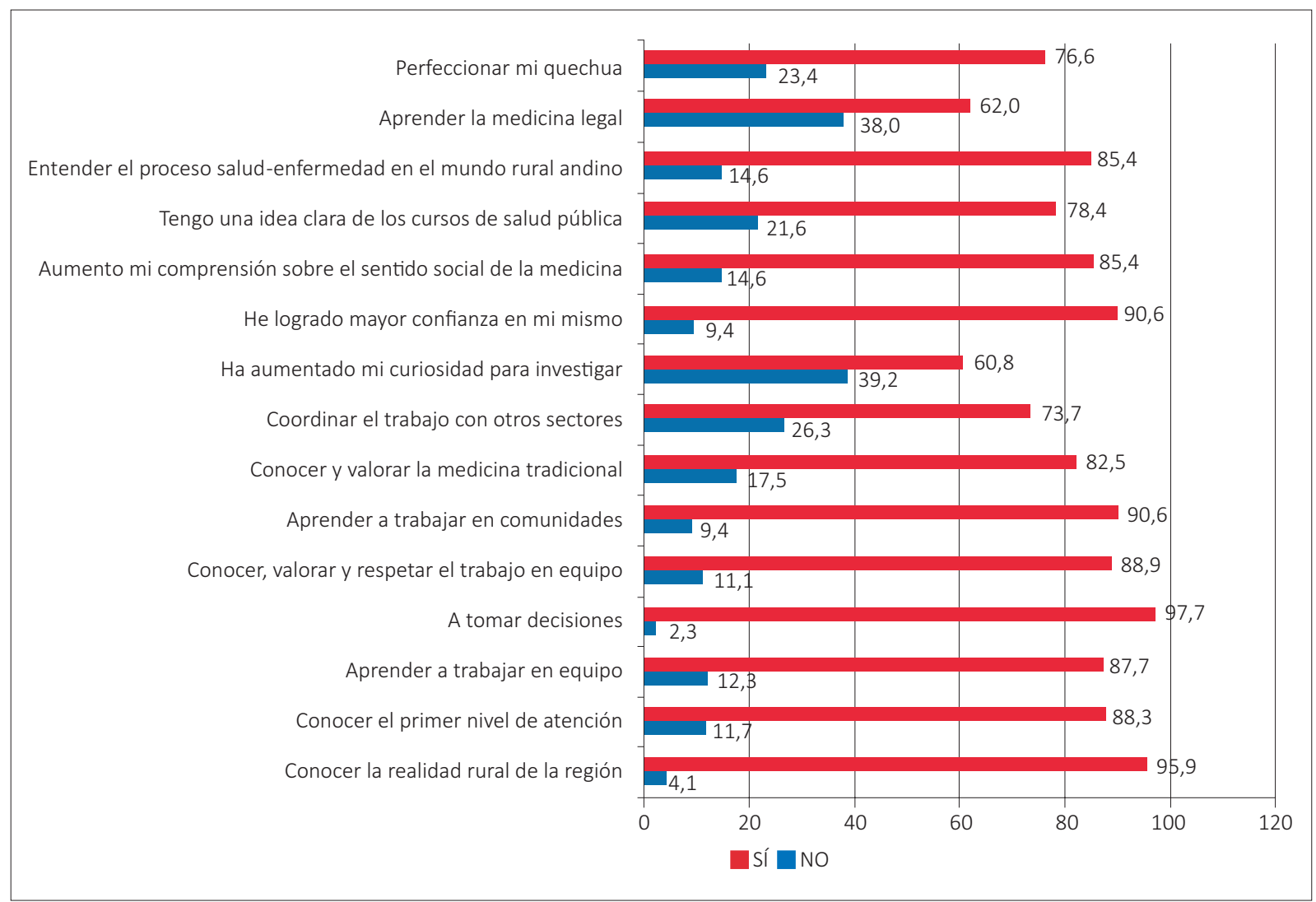

Figura 2. Aspectos de la formación médica en el internado rural por parte de los egresados FMH-UNSAAC.

\section{DISCUSIÓN}

Considerando el problema y los objetivos propuestos, que buscan describir la mejora de las condiciones de salud y la calidad de vida de nuestras poblaciones, con un trabajo conjunto de la universidad con los servicios de salud a través de la IDA y comunidad, concluimos que la IDA es la estrategia que nos ayuda a mejorar la formación y el desempeño de los futuros médicos generales.

La mayoría de las facultades y escuelas de medicina peruanas y latinoamericanas mantienen sus ámbitos de enseñanza-aprendizaje en las aulas, laboratorios universitarios y en los servicios de salud, que generalmente son los hospitales del tercer nivel y los institutos especializados, en desmedro de la enseñanza en los servicios del primer nivel de atención y menos aun en la comunidad. Este modelo es el reflejo de la práctica médica dominante, que ha centrado su actividad en los hospitales, donde se realiza el proceso de enseñanza-aprendizaje. Esto a su vez es una consecuencia de los actuales sistemas de salud, cuya preocupación está centrada solo en brindar servicios de salud curativos a determinadas minorías centralizadas en las grandes ciudades. En ese sentido, la universidad también mantiene un rol pasivo y acrítico por el sistema de salud, legitimando acciones que no tienen coherencia con la realidad de salud de la población del país.

Considerando las necesidades reales de nuestra población eminentemente rural y dispersa en un gran ámbito geográfico accidentado, con variados pisos ecológicos, con precarias vías de comunicación, patrones culturales milenarios bien arraigados y catalogada oficialmente como de extrema pobreza, con graves problemas de salud-enfermedad, la Facultad de Medicina Humana de la UNSAAC planteó, a fines de los años 70 y comienzo de los 80, brindar una formación médica que responda a dichas necesidades, experiencia que se viene desarrollando hasta la fecha.
Finalmente, en la encuesta realizada a los alumnos que realizaron el internado rural, estos califican a la experiencia como excelente y buena en $81 \%$, que les ha permitido ampliar sus conocimientos prácticos y les ha dado la oportunidad de conocer el sector rural y un servicio de salud del primer nivel, a tomar decisiones, a trabajar en equipo, conocer y valorar la medicina tradicional, entre otros.

Concluimos que la IDA contribuye a mejorar la situación de salud de las comunidades intervenidas, la calidad de los servicios en los establecimientos de salud, e influye en la formación y el desempeño del futuro médico cirujano.

\section{REFERENCIAS BIBLIOGRÁFICAS}

1. Vidal C, Quiñones J. Integración Docente Asistencial. Educ Med Salud. 1986;20(4):458-65.

2. Declaración de Alma-Ata. En: Alma-Ata 1978: Atención primaria de salud. Informe de la Conferencia Internacional sobre Atención Primaria de Salud, septiembre 6-12 de 1978; Alma-Ata, URSS. Ginebra: Organización Mundial de la Salud; 1978. 
3. Arteaga A, Álvarez A, Cabrera N, Toledo A. La integración docente, asistencial e investigativa en la Atención Primaria de Salud. Rev Cubana Med Gen Integr. 2010;26(2):350-9.

4. Universidad Nacional San Antonio de Abad del Cusco. Cusco: Universidad Nacional San Antonio de Abad del Cusco; 2017 [citado 22 marzo 2017]. Disponible en: http://mh.unsaac.edu.pe/ historia.php.
5. Farfán Cantero R. Odisea de la educación médica en Cusco. SITUA. 2002;10(20).

6. Farfán R, Saco S. Integración Universidad Servicios de Salud-Comunidad en la Facultad de Medicina Humana de La Universidad Nacional de San Antonio Abad del Cusco. SITUA. 1998;6(11).

7. Brito P, Lazo O, Lip C. El Trabajo Médico en el Perú. Lima: OPS-Universidad Particular Cayetano Heredia; 1989.
8. Alarcón J, Reyes N, Piscoya J. Situación de trabajo y características socioeconómicas del médico en Lima Metropolitana. Lima: OPS-Colegio Médico del Perú; 1989. 